\title{
Marco Di Palma, «Un soin que je me dois»: self- regarding virtues in Rousseau's Dialogues
}

\section{Stefania Carli}

\section{(2) OpenEdition}

12 Journals

\section{Edizione digitale}

URL: http://journals.openedition.org/studifrancesi/30328

DOI: 10.4000/studifrancesi.30328

ISSN: 2421-5856

\section{Editore}

Rosenberg \& Sellier

\section{Edizione cartacea}

Data di pubblicazione: 1 avril 2006

Paginazione: 161

ISSN: 0039-2944

\section{Notizia bibliografica digitale}

Stefania Carli, «Marco Di Palma, «Un soin que je me dois»: self-regarding virtues in Rousseau's

Dialogues», Studi Francesi [Online], 148 (XLX | I) | 2006, online dal 30 novembre 2015, consultato il 18 avril 2021. URL: http://journals.openedition.org/studifrancesi/30328 ; DOI: https://doi.org/10.4000/ studifrancesi.30328

Questo documento è stato generato automaticamente il 18 avril 2021.

\section{(c) (1)}

Studi Francesi è distribuita con Licenza Creative Commons Attribuzione - Non commerciale - Non opere derivate 4.0 Internazionale. 


\title{
Marco Di Palma, «Un soin que je me dois»: self-regarding virtues in Rousseau's Dialogues
}

\author{
Stefania Carli
}

\section{NOTIZIA}

MARCO DI PALMA, «Un soin que je me dois»: self-regarding virtues in Rousseau's Dialogues, «French Studies», vol. 58, n. 1 (2004), pp. 15-27.

1 L'articolo si propone di analizzare le idee espresse da Jean-Jacques Rousseau nei suoi Dialogues. L'intento di Marco di Palma è quello di individuare una maggiore continuità tra il pensiero presente in quest'opera e gli altri scritti dell'autore. L'interesse dello studioso è incentrato principalmente sull'attenzione che Rousseau rivolge all'analisi del proprio io: si tratta di uno sguardo introspettivo fondamentale per individuare il modo migliore per raggiungere la felicità.

Di Palma si prefigge tre obiettivi: il primo è quello di avanzare una nuova interpretazione sullo status etico e politico dei Dialogues, mettendo in relazione quest'opera con gli altri scritti di Rousseau. Dall'attenta analisi condotta dallo studioso, si deduce che i Dialogues attestano e confermano l'importanza che l'autore dà alla morale: per Rousseau essa è collegata alla ricerca personale che viene condotta da ciascuno per il raggiungimento del proprio benessere ed è strettamente associata all'idea di virtù.

3 In secondo luogo, Di Palma affronta e rivaluta le teorie precedentemente avanzate da Jean Starobinski, che aveva visto nella riforma intellettuale e morale presentata dall'autore dei Dialogues, una falsa ascesa verso un totale "divestment of the will". Al contrario, Di Palma sostiene che questa sorta di riforma sia da intendere come uno sforzo per conquistare una maggiore libertà personale. Egli afferma che «The Dialogues convey no aesthetic retreat from Rousseau's earlier philosophy, as Starobinski argues, 
but rather its vindication as an ongoing task for moral liberty». A questa libertà si arriverebbe attraverso un'esistenza vissuta in accordo e in sintonia con la natura, ma anche in armonia con le virtù intellettuali e le leggi morali, che per Rousseau sono la giustizia, la semplicità, la temperanza, la prudenza e la saggezza. Grazie al suo alter ego Jean-Jacques, l'autore del Dialogues evidenzia la pratica di una disciplina allo stesso tempo razionale e spirituale che si basa essenzialmente sulla ricerca della virtù. Obiettivo principale di Rousseau è infatti quello di arrivare a vivere un'esistenza semplice e laboriosa che possa rendere, a se stesso come a qualsiasi uomo, la giusta felicità.

L'ultimo obiettivo che si pone l'articolo di Di Palma è di mostrare come la ricerca di un bene privato e personale nel contesto stoico ed epicureo definisca le possibilità morali dell'opera di Rousseau. Come i praticanti delle due discipline filosofiche, anche l'autore dei Dialogues è convinto che il cosiddetto bonheur non si trovi in uno status sociale elevato, bensì nella semplicità e nella soddisfazione interiore, ottenuta grazie alla buona coscienza di ognuno. Secondo Rousseau la "faiblesse de l'âme" corrompe e rende l'uomo schiavo in quanto lo fa dipendere da bisogni inutili e falsi. Il modo individuato dall'autore per raggiungere la felicità sta nel saper equilibrare le proprie necessità e le possibilità che si hanno di soddisfarle. Ecco allora che i Dialogues propongono in definitiva l'ideale della mediocritas, vale a dire di quello stato semplice e tranquillo nel quale ogni uomo può ritrovare la propria serenità senza essere distratto da continue illusioni. 